\title{
A Brief Survey on the Characteristics of Recent Virtual Exhibitions
}

\author{
Keywords \\ Digital Space, Tangible Space, Virtual Exhibitions, 360 photography, 3D modelling
}

The Covid-19 pandemic and the social restriction measures that ensued have had a decisive impact on museum activities over the past two years. Research by the International Council of Museums (ICOM) indicates that about $95 \%$ of worldwide museums were closed between April and May 2020 , a figure that falls to $27 \%$ in the same period in 2021. Given this scenario, there has been a notable increase in digital communications and activities of these institutions, either by making collections and exhibitions available online, managing social media, holding live events and teaching programs or by producing newsletters and podcasts. While such surveys are essential, it is also necessary to complement them with qualitative research into the ways in which these activities are being carried out. This paper will examine how a specific kind of digital activity is being developed by the museum sector, namely, the virtual exhibitions. Characterized mainly by the three-dimensionality and the immersion of the interactor, these virtual exhibition spaces carry with them both the complexity that constitutes the idea of a virtual museum and the structural deficiencies that affect cultural institutions and, in particular, their sectors of Information and Communication Technology (ICTs). Thus, two criteria were chosen to characterize these exhibits, namely, the technologies commonly used in their conception and the relationship they establish or do not establish with a tangible space. To this end, recent cases of spaces built through the use of $360^{\circ}$ photography and 3D modeling have been selected and analyzed as to highlight the distinctive features of these technologies and how they instantiate different relationships between elements of the digital and the tangible. This analysis bring into consideration specific elements of these exhibitions, such as their interfaces and the modes of displacement and visualization they allow, the quality of the reproduction of the artworks and the possibility of accessing complementary information and media about them, the multiplicity of the points of view, their compatibility with certain devices, among others. Finally, we will reflect on the way in which these virtual exhibitions replicate, augment or dismiss physical spaces for their conception. Instead of the usual opposition between virtual and actual, such spaces express the possibility of complementation between elements restricted to the tangible or the digital - complementation, needless to say, that does not always take place. Thus, virtual exhibitions are important vectors to reflect on the advantages and difficulties that digital technologies pose for the museum sector. 